\title{
Exact Controllability of the Time Discrete Wave Equation: a Multiplier Approach
}

\author{
Xu Zhang ${ }^{1}$, Chuang Zheng ${ }^{2}$, and Enrique Zuazua ${ }^{3}$ \\ 1 Academy of Mathematics and Systems Sciences, Chinese Academy of Sciences, \\ CN-100080 Beijing, China xuzhang@amss.ac.cn \\ 2 School of Mathematical Sciences, Beijing Normal University, CN-100875 Beijing, \\ China chuang.zheng@bnu.edu.cn \\ 3 Basque Center for Applied Mathematics, Bizkaia Technology Park, Building 500, \\ ES-48160 Derio, Basque Country, Spain zuazua@bcamath.org, \\ http://www.bcamath.org/zuazua/
}

Summary. In this paper we summarize our recent results on the exact boundary controllability of a trapezoidal time discrete wave equation in a bounded domain. It is shown that the projection of the solution in an appropriate space in which the high frequencies have been filtered is exactly controllable with uniformly bounded controls (with respect to the time-step). By classical duality arguments, the problem is reduced to a boundary observability inequality for a time-discrete wave equation. Using multiplier techniques the uniform observability property is proved in a class of filtered initial data. The optimality of the filtering parameter is also analyzed.

Key words: Exact controllability, observability, time discretization, wave equation, multiplier technique, filtering.

\section{Introduction}

Let $\Omega$ be an open bounded domain in $\mathbb{R}^{d}\left(d \in \mathbb{N}^{*}\right)$ with $C^{2}$ boundary $\Gamma$. Let $T>0$ be a given time duration. We consider the following wave equation with a state $y=y(x, t)$ and a controller $u=u(x, t)$ acting on the nonempty subset $\Gamma_{0}$ of the boundary $\Gamma=\partial \Omega$ :

$$
\begin{cases}y_{t t}-\Delta y=0 & \text { in }(0, T) \times \Omega, \\ y=u 1_{\Gamma_{0}} & \text { on }(0, T) \times \Gamma, \\ y(0)=y_{0}, \quad y_{t}(0)=y_{1} & \text { in } \Omega .\end{cases}
$$

Here $1_{\Gamma_{0}}$ is the characteristic function of the set $\Gamma_{0}$.

This paper is devoted to analyze whether the known controllability results for (1) can be recovered as a consequence of similar results for the time-discrete versions. This kind of problems has been the object of intensive research in 
the past few years but mainly in the context of space semi-discretizations. In the present paper we summarize the main results by the authors [14] in the time discrete case. This issue is of interest from a numerical analysis point of view but also in what concerns the link between the control properties of time continuous and time-discrete distributed parameter systems. The topic of numerical approximation of boundary controls for wave equations was initiated by R. Glowinski, J.-L. Lions and coworkers (see, for instance, $[3,14]$ ) and has motivated intensive research (we refer to [17] for a survey).

The exact controllability of (1) requires that the subset $\Gamma_{0}$ of the boundary fulfills some geometric conditions. It holds, in particular, for those subsets that are obtained through the multiplier method. More precisely, fix some $x_{0} \in \mathbb{R}^{d}$, and put

$$
\left\{\begin{array}{l}
R \triangleq \max _{x \in \Omega}\left|x-x_{0}\right|, \\
\Gamma_{0} \triangleq\left\{x \in \Gamma \mid\left(x-x_{0}\right) \cdot \nu(x)>0\right\},
\end{array}\right.
$$

where $\nu(x)$ is the unit outward normal vector of $\Omega$ at $x \in \Gamma$. For these subsets $\Gamma_{0}$ the exact controllability property of (1) holds provided $T>2 R$.

To be more precise, the following exact controllability result for (1) is well known (see [6]): For any $\left(y_{0}, y_{1}\right) \in L^{2}(\Omega) \times H^{-1}(\Omega)$, there exists a control $u \in L^{2}\left((0, T) \times \Gamma_{0}\right)$ such that the solution $y=y(t, x)$ of $(1)$, defined by the classical transposition method, satisfies

$$
y(T)=y_{t}(T)=0 \quad \text { in } \Omega .
$$

By classical duality arguments [6], the above controllability property is equivalent to a (boundary) observability one of the following uncontrolled wave equation:

$$
\begin{cases}\varphi_{t t}-\Delta \varphi=0, & \text { in }(0, T) \times \Omega \\ \varphi=0 & \text { on }(0, T) \times \Gamma \\ \varphi(T)=\varphi_{0}, \quad \varphi_{t}(T)=\varphi_{1}, & \text { in } \Omega,\end{cases}
$$

i.e., to the fact that solutions of (4) satisfy

$$
E(0) \leq C \int_{0}^{T} \int_{\Gamma_{0}}\left\|\frac{\partial \varphi}{\partial \nu}\right\|^{2} d \Gamma_{0} d t, \quad \forall\left(\varphi_{0}, \varphi_{1}\right) \in H_{0}^{1}(\Omega) \times L^{2}(\Omega) .
$$

Here and thereafter, we will use $C$ to denote a generic positive constant (depending only on $T, \Omega$ and $\Gamma_{0}$ ) which may vary from line to line. On the other hand, $E(0)$ stands for the energy $E(t)$ of (4) at $t=0$, with

$$
E(t)=\frac{1}{2} \int_{\Omega}\left[\left|\varphi_{t}(t, x)\right|^{2}+|\nabla \varphi(t, x)|^{2}\right] d x,
$$

which remains constant, i.e.

$$
E(t)=E(0), \quad \forall t \in[0, T] .
$$


The inequality (5) can be proved by several methods including multiplier techniques [6], microlocal analysis [1] and Carleman inequalities [13]. In the particular case of subset $\Gamma_{0}$ as above and $T>2 R$, the inequality (5) can be proved easily by the method of multipliers [6] that in the present paper we adapt to time-discrete equations.

Note, however, that the subsets $\Gamma_{0}$ of the boundary and the values of the minimal control time obtained in this way are not optimal. The obtention of optimal control subsets and times requires the use of methods of geometric optics (see [1]).

In this paper, we analyze time semi-discretization schemes for the systems (1) and (4). We are thus replacing the continuous dynamics (1) and (4) by time-discrete ones and analyze their controllability/observability properties. Here we take the point of view of numerical analysis and, therefore, we analyze the limit behavior as the time-step tends to zero.

More precisely, we set the time step $h$ by $h=T / K$, where $K>1$ is a given odd integer. Denote by $y^{k}$ and $u^{k}$ respectively the approximations of the solution $y$ and the control $u$ of (1) at time $t_{k}=k h$ for any $k=0, \ldots, K$. We then introduce the following trapezoidal time semi-discretization of (1):

$$
\begin{cases}\frac{y^{k+1}+y^{k-1}-2 y^{k}}{h^{2}}-\Delta\left(\frac{y^{k+1}+y^{k-1}}{2}\right)=0, & \text { in } \Omega, k=1, \ldots, K-1, \\ y^{k}=u^{k} 1_{\Gamma_{0}}, & \text { on } \Gamma, k=0, \ldots, K, \\ y^{0}=y_{0}, \quad y^{1}=y_{0}+h y_{1}, & \text { in } \Omega .\end{cases}
$$

Here $\left(y_{0}, y_{1}\right) \in L^{2}(\Omega) \times H^{-1}(\Omega)$ are the data in the system (1). We refer to Theorem 1 below for the well-posedness of the system (1) by means of a transposition method.

The controllability problem for the system (7) is formulated as follows: For any $\left(y_{0}, y_{1}\right) \in L^{2}(\Omega) \times H^{-1}(\Omega)$, to find a control $\left\{u^{k} \in L^{2}\left(\Gamma_{0}\right)\right\}_{k=1, \ldots, K-1}$ such that the solution $\left\{y^{k}\right\}_{k=0, \ldots, K}$ of (7) satisfies:

$$
y^{K-1}=y^{K}=0 \quad \text { in } \Omega .
$$

Note that (8) is equivalent to the condition $y^{K-1}=\left(y^{K}-y^{K-1}\right) / h=0$ that is a natural discrete version of (3).

As in the context of the above continuous wave equation, we also consider the uncontrolled system

$$
\begin{cases}\frac{\varphi^{k+1}+\varphi^{k-1}-2 \varphi^{k}}{h^{2}}-\Delta\left(\frac{\varphi^{k+1}+\varphi^{k-1}}{2}\right)=0, & \text { in } \Omega, k=1, \ldots, K-1, \\ \varphi^{k}=0, & \text { on } \Gamma, k=0, \ldots, K \\ \varphi^{K}=\varphi_{0}^{h}+h \varphi_{1}^{h}, \quad \varphi^{K-1}=\varphi_{0}^{h}, & \text { in } \Omega,\end{cases}
$$

where $\left(\varphi_{0}^{h}, \varphi_{1}^{h}\right) \in\left(H_{0}^{1}(\Omega)\right)^{2}$. In particular, to guarantee the convergence of the solutions of (9) towards those of (4) one considers convergent data such 
that

$$
\left\{\begin{array}{ll}
\varphi_{0}^{h} \rightarrow \varphi_{0} & \text { strongly in } H_{0}^{1}(\Omega), \\
\varphi_{1}^{h} \rightarrow \varphi_{1} & \text { strongly in } L^{2}(\Omega), \\
h \varphi_{1}^{h} \rightarrow \varphi_{1} & \text { is bounded in } H_{0}^{1}(\Omega),
\end{array} \quad \text { as } K \rightarrow \infty(\text { or } h \rightarrow 0)\right.
$$

Obviously, because of the density of $H_{0}^{1}(\Omega)$ in $L^{2}(\Omega)$, this choice is always possible.

Remark 1. Note that the choice of the values of $\varphi^{K}$ and $\varphi^{K-1}$ in (9) is motivated by the definition of the solution of the time-discrete non-homogenous problem (7) in the sense of transposition (see Definition 1).

The energy of the system (9) is given by

$$
E_{h}^{k} \triangleq \frac{1}{2} \int_{\Omega}\left(\left\|\frac{\varphi^{k+1}-\varphi^{k}}{h}\right\|^{2}+\frac{\left|\nabla \varphi^{k+1}\right|^{2}+\left|\nabla \varphi^{k}\right|^{2}}{2}\right) d x, \quad k=0, \ldots, K-1,
$$

which is a discrete counterpart of the continuous energy $E$ in (6). It is easy to show that $E_{h}^{k}$ is conserved in the discrete time variable $k=0, \ldots, K-1$. Consequently, the scheme under consideration is stable and its convergence (in the classical sense of numerical analysis) is guaranteed (in the finite-energy space $H_{0}^{1}(\Omega) \times L^{2}(\Omega)$ of the system $\left.(4)\right)$.

By means of classical duality arguments, it is easy to show that the above controllability property (8) is equivalent to the following boundary observability property for solutions $\left\{\varphi^{k}\right\}_{k=0, \ldots, K}$ of $(9)$ :

$$
E_{h}^{0} \leq C h \sum_{k=1}^{K-1} \int_{\Gamma_{0}}\left|\frac{\partial}{\partial \nu}\left(\frac{\varphi^{k+1}+\varphi^{k-1}}{2}\right)\right|^{2} d \Gamma_{0}, \quad \forall\left(\varphi_{0}^{h}, \varphi_{1}^{h}\right) \in\left(H_{0}^{1}(\Omega)\right)^{2} .
$$

As we mentioned above, the controllability/observability properties of numerical approximation schemes for the wave equation have been the object of intensive studies. However, most analytical results concern the case of space semi-discretizations (see [17] and the references cited therein). In practical applications, fully discrete schemes need to be used. The most typical example is the classical fully-discrete central scheme which converges under a suitable CFL condition $[3,4,11]$. However, in the present setting in which the space Laplacian $\Delta$ is kept continuous, without discretizing it, this scheme is unsuitable since it is unstable. Indeed, it is easy to see that the scheme

$$
\frac{\varphi^{k+1}+\varphi^{k-1}-2 \varphi^{k}}{h^{2}}-\Delta \varphi^{k}=0
$$

is unstable since $-\Delta$, with homogenous Dirichlet conditions, is a positive selfadjoint operator with an infinite sequence of eigenvalues $\left\{\mu_{j}^{2}\right\}_{j \geq 1}$ tending to infinity. The stability of (12) would be equivalent to the stability of the scheme 


$$
\frac{\varphi^{k+1}+\varphi^{k-1}-2 \varphi^{k}}{h^{2}}+\mu_{j}^{2} \varphi^{k}=0
$$

for all values of $\mu_{j}^{2}, j \geq 1$. This stability property fails clearly, regardless how small $h$ is, when $\mu_{j}^{2}$ is large enough. Hence, we choose the trapezoidal scheme (9) for the time-discrete problem, which is stable (due to the property of conservation of energy), as mentioned before.

Let us now return to the analysis of (7) and (9). Noting that the spaces in which the solutions of these systems evolve are infinite dimensional while the number of time-steps is finite, it is easy to conclude that: For any given $h>0$, the inequality (11) fails and the system (7) is not exactly controllable. Accordingly, to make the observability inequality possible, one has to restrict the class of solutions of the adjoint system (9) under consideration by filtering the high frequency components. Similarly, since the property of exact controllability of the system (7) fails, the final requirement (8) has to be relaxed by considering only low frequency projections of the solutions. Controlling such a projection can be viewed as a partial controllability problem. This filtering method has been applied successfully in the context of controllability of time discrete heat equations in [15] and space semi-discretization schemes for wave equations in $[5,16,17]$.

In this paper, we sketch the discrete version of the classical multiplier approach developed in [14] which allows to derive the uniform observability estimate (with respect to the time step $h$ ) for the system (9) with initial data in a suitable filtered space, which, in turn, by duality, implies the partial controllability of (7), uniformly on $h$.

As in the continuous case, the multiplier technique applies mainly to the case when the controller/observer $\Gamma_{0}$ is given in (2) and some variants [9], but does not work when $\left(T, \Omega, \Gamma_{0}\right)$ is assumed to satisfy the sharp Geometric Control Condition (GCC) in [1]. As we shall see, the main advantage of our multiplier approach is that the filtering parameter we use has the optimal scaling in what concerns the frequency of observed/controlled solutions with respect to $h$.

The rest of the paper is organized as follows. In Section 2 we state the main results, i.e., the uniform controllability and observability of the systems (7) and (9) after filtering, respectively. In Section 3 we give a heuristic explanation of the necessity of the filtering analyzing the bicharacteristic rays and the group velocity. The key ingredients in the proof of the uniform observability results will be sketched in Sections 4 and 5. Finally, in Section 6, we shall briefly discuss some open problems and closely related issues.

\section{Main Results}

We begin with the well-posedness of the system (7). For this purpose, for any $\left\{f^{k} \in L^{2}(\Omega)\right\}_{k=1, \ldots, K-1}$, and any $\left\{g^{k} \in H_{0}^{1}(\Omega)\right\}_{k=1, \ldots, K}$ with $g^{1}=g^{K}=0$, we consider the following adjoint problem of the system (7): 


$$
\begin{cases}\frac{\zeta^{k+1}+\zeta^{k-1}-2 \zeta^{k}}{h^{2}}-\Delta\left(\frac{\zeta^{k+1}+\zeta^{k-1}}{2}\right) & \\ \quad=f_{k}+\frac{g^{k+1}-g^{k}}{h}, & \text { in } \Omega, k=1, \ldots, K-1, \\ \zeta^{k}=0, & \text { on } \Gamma, k=0, \ldots, K \\ \zeta^{K}=\zeta^{K-1}=0, & \text { in } \Omega .\end{cases}
$$

It is easy to see that (13) admits a unique solution $\left\{\zeta^{k} \in H_{0}^{1}(\Omega)\right\}_{k=0, \ldots, K}$. Moreover, this solution has the regularity $\frac{\partial}{\partial \nu}\left(\frac{\zeta^{k+1}+\zeta^{k-1}}{2}\right) \in L^{2}(\Gamma)$ for $k=$ $1, \ldots, K-1$.

Put

$$
\begin{aligned}
& \mathcal{H}=\left\{\left\{y^{k}\right\}_{k=0, \ldots, K} \mid y^{i+1}+y^{i-1} \in L^{2}(\Omega) \text { for } i=1, \ldots, K-1,\right. \\
&\left.\frac{y^{j+1}-y^{j}}{h}+\frac{y^{j-1}-y^{j-2}}{h} \in H^{-1}(\Omega) \text { for } j=2, \ldots, K-1\right\} .
\end{aligned}
$$

We introduce the following:

Definition 1. $\left\{y^{k}\right\}_{k=0, \ldots, K} \in \mathcal{H}$ is said to be a solution of (7), in the sense of transposition, if $y^{0}=y_{0}, y^{1}=y_{0}+h y_{1}$, and for any $\left\{f^{k} \in L^{2}(\Omega)\right\}_{k=1, \ldots, K-1}$, and $\left\{g^{k} \in H_{0}^{1}(\Omega)\right\}_{k=1, \ldots, K}$ with $g^{1}=g^{K}=0$, it holds

$$
\begin{aligned}
& h \sum_{k=1}^{K-1} \int_{\Omega} f^{k} \frac{y^{k+1}+y^{k-1}}{2} d x \\
&-h \sum_{k=2}^{K-1}\left\langle g^{k}, \frac{y^{k+1}-y^{k}}{2 h}+\frac{y^{k-1}-y^{k-2}}{2 h}\right\rangle_{H_{0}^{1}(\Omega), H^{-1}(\Omega)} \\
&=\left\langle\zeta^{0}, y_{1}\right\rangle_{H_{0}^{1}(\Omega), H^{-1}(\Omega)}-\int_{\Omega} \frac{\zeta^{1}-\zeta^{0}}{h} y_{0} d x \\
& \quad-h \sum_{k=1}^{K-1} \int_{\Gamma_{0}} \frac{\partial}{\partial \nu}\left(\frac{\zeta^{k+1}+\zeta^{k-1}}{2}\right) u^{k} d \Gamma_{0},
\end{aligned}
$$

where $\left\{\zeta^{k} \in H_{0}^{1}(\Omega)\right\}_{k=0, \ldots, K}$ is the solution of (13).

The above definition can be viewed as a discrete version of the classical transposition approach [6]. It is motivated by the following observation: When the control $\left\{u^{k}\right\}_{k=0, \ldots, K}$ and the initial data $\left(y^{0}, y^{1}\right)$ are sufficiently smooth, multiplying both sides of (13) by $\left(y^{k+1}+y^{k-1}\right) / 2$, integrating the resulting identity in $\Omega$ and summing it for $k=1, \ldots, K-1$, one obtains (15).

The well-posedness of the system (7) is stated as follows:

Theorem 1. Assume $\left(y_{0}, y_{1}\right) \in L^{2}(\Omega) \times H^{-1}(\Omega)$ and $\left\{u^{k} \in L^{2}\left(\Gamma_{0}\right)\right\}_{k=1, \ldots, K-1}$. Then the system (7) admits one and only one solution $\left\{y^{k}\right\}_{k=0, \ldots, K} \in \mathcal{H}$ in 
the sense of Definition 1. Moreover, $\left(y^{2 \ell}, \frac{y^{2 \ell+1}-y^{2 \ell}}{h}\right) \in L^{2}(\Omega) \times H^{-1}(\Omega)$ for $\ell=0,1, \ldots,\left[\frac{K}{2}\right]$, and

$$
\begin{aligned}
\max _{\ell=0,1, \ldots,\left[\frac{K}{2}\right]}\left\|\left(y^{2 \ell}, \frac{y^{2 \ell+1}-y^{2 \ell}}{h}\right)\right\|_{L^{2}(\Omega) \times H^{-1}(\Omega)}^{2} & \\
& \leq C\left(\left\|\left(y_{0}, y_{1}\right)\right\|_{L^{2}(\Omega) \times H^{-1}(\Omega)}^{2}+h \sum_{k=1}^{K-1}\left\|u^{k}\right\|_{L^{2}\left(\Gamma_{0}\right)}^{2}\right) .
\end{aligned}
$$

We refer to [14] for the proof of Theorem 1 by means of a discrete multiplier approach.

Next, assume $\left\{\Phi_{j}\right\}_{j \geq 1} \subset H_{0}^{1}(\Omega)$ to be an orthonormal basis of $L^{2}(\Omega)$ consisting of the eigenvectors (with eigenvalues $\left\{\mu_{j}^{2}\right\}_{j \geq 1}$ ) of the Dirichlet Laplacian:

$$
\begin{cases}-\Delta \Phi_{j}=\mu_{j}^{2} \Phi_{j}, & \text { in } \Omega \\ \Phi_{j}=0, & \text { on } \Gamma .\end{cases}
$$

For any $s>0$, we set

$$
\begin{aligned}
& \mathcal{C}_{1, s}=\left\{f(x) \mid f(x)=\sum_{\mu_{j}^{2}<s} a_{j} \Phi_{j}(x), a_{j} \in \mathbb{C}\right\} \subset H_{0}^{1}(\Omega), \\
& \mathcal{C}_{0, s}=\left\{g(x) \mid g(x)=\sum_{\mu_{j}^{2}<s} b_{j} \Phi_{j}(x), b_{j} \in \mathbb{C}\right\} \subset L^{2}(\Omega),
\end{aligned}
$$

and

$$
\mathcal{C}_{-1, s}=\left\{z(x) \mid z(x)=\sum_{\mu_{j}^{2}<s} c_{j} \Phi_{j}(x), c_{j} \in \mathbb{C}\right\} \subset H^{-1}(\Omega),
$$

subspaces of $H_{0}^{1}(\Omega), L^{2}(\Omega)$ and $H^{-1}(\Omega)$, respectively, with the induced topologies. It is clear that $\bigcup_{k=1}^{\infty} \mathcal{C}_{1, k}$ is dense in $H_{0}^{1}(\Omega)$, and the same can be said for $\bigcup_{k=1}^{\infty} \mathcal{C}_{0, k}$ in $L^{2}(\Omega)$ and $\bigcup_{k=1}^{\infty} \mathcal{C}_{-1, k}$ in $H^{-1}(\Omega)$. Denote by $\pi_{1, s}$, $\pi_{0, s}$ and $\pi_{-1, s}$ the projection operators from $H_{0}^{1}(\Omega), L^{2}(\Omega)$ and $H^{-1}(\Omega)$ to $\mathcal{C}_{1, s}, \mathcal{C}_{0, s}$ and $\mathcal{C}_{-1, s}$, respectively.

Our main results are stated as follows:

Theorem 2. Let $T>2 R$. Then there exist three constants $h_{0}>0, \delta>0$ and $C>0$, depending only on $T, R$ and the dimension $d$, such that for all $\left(\varphi_{0}, \varphi_{1}\right) \in \mathcal{C}_{1, \delta h^{-2}} \times \mathcal{C}_{0, \delta h^{-2}}$, the corresponding solution $\left\{\varphi^{k}\right\}_{k=0, \ldots, K}$ of (9) satisfies

$$
E_{h}^{0} \leq C h \sum_{k=1}^{K-1} \int_{\Gamma_{0}}\left\|\frac{\partial}{\partial \nu}\left(\frac{\varphi^{k+1}+\varphi^{k-1}}{2}\right)\right\|^{2} d \Gamma_{0}, \quad \forall h \in\left(0, h_{0}\right] .
$$


Remark 2. We refer to (38) for the exact form of $\delta$, which depends only on $d$, $T$ and $R$. In particular, it indicates that $\delta$ decreases as $T$ decreases. This is natural since, as $T$ decreases, less and less time-step iterations are involved in the system (9) and, consequently, less Fourier components of the solutions may be observed. Further, $\delta$ tends to zero as $T$ tends to $2 R$. This is natural too since our proof of (20) is based on the method of multipliers which works at the continuous level for all $T>2 R$ but that, at the time-discrete level, due to the added dispersive effects, may hardly work when $T$ is very close to $2 R$, except if the filtering is strong enough.

Remark 3. The problem considered in this paper could have been addressed, in $1-d$, using discrete Ingham inequalities as those in [8]. When doing that, one would get similar results. In [2] the problem of observability of time-discrete linear conservative systems is addressed in an abstract context including wave, plate and Schrödinger equations. The techniques employed in [2] are inspired in those in [10] based on resolvent estimates, which allow to derive, in a systematic way, observability results for time-discrete systems as consequences of those that are by now well-known for time-continuous ones. The results in [2] can be applied to the time-discrete wave equation considered in this article. The main drawback of the results in [2] is that the observability time one gets seems to be far from the expected optimal one. Another different approach, which gives weaker results, is viewing (by extension to continuous time) the solutions of (9) as perturbed solutions of the continuous conservative wave equation (4). Absorbing the remainder terms then requires stronger filtering than the multiplier method.

Remark 4. As shown in [14], the order $h^{-2}$ of the filtering parameter (in Theorem 2) is optimal. This corresponds precisely to filtering numerical solutions whose wave length is of the order of the mesh-size $h$, for which resonance phenomena may arise. However, our analysis in the next section indicates that the inequality (20) may hold within the class $\mathcal{C}_{1, \delta h^{-2}} \times \mathcal{C}_{0, \delta h^{-2}}$ for any $\delta>0$. This can be proved to hold by applying the abstract results in [2] to the present problem. The multiplier method we develop here needs to impose a smallness condition on $\delta$. It is an interesting open problem to see if the multiplier method can be adapted to deal with arbitrarily large values of $\delta$. But it is well known, even at the continuous level, that the method of multipliers is often unable to yield observability results that can be obtained by other ways.

As a consequence of the partial observability result in Theorem 2 , by duality, we can derive the following uniform partial controllability result:

Theorem 3. Let $T, h_{0}$ and $\delta$ be given as in Theorem 2. Then for any $h \in$ $\left(0, h_{0}\right]$ and any $\left(y^{0}, \frac{y^{1}-y^{0}}{h}\right) \in L^{2}(\Omega) \times H^{-1}(\Omega)$, there exists a control $\left\{u^{k} \in\right.$ $\left.L^{2}\left(\Gamma_{0}\right)\right\}_{k=0, \ldots, K}$ such that the solution of (7) satisfies the following:

(i) It holds 


$$
\pi_{0, \delta h^{-2}} y^{K-1}=\pi_{-1, \delta h^{-2}}\left(\frac{y^{K}-y^{K-1}}{h}\right)=0 \quad \text { in } \Omega
$$

(ii) There exists a constant $C>0$, independent of $h, y^{0}$ and $y^{1}$, such that

$$
h \sum_{k=1}^{K-1} \int_{\Gamma_{0}}\left\|\frac{u^{k+1}+u^{k-1}}{2}\right\|^{2} d \Gamma_{0} \leq C\left\|\left(y^{0}, \frac{y^{1}-y^{0}}{h}\right)\right\|_{L^{2}(\Omega) \times H^{-1}(\Omega)}^{2} ;
$$

(iii) When $h \rightarrow 0$,

$$
U_{h} \triangleq \sum_{k=1}^{K-1} u^{k}(x) 1_{[k h,(k+1) h)}(t) \longrightarrow u \quad \text { strongly in } L^{2}\left((0, T) \times \Gamma_{0}\right),
$$

where $u$ is a control of the system (1), fulfilling (3);

(iv) When $h \rightarrow 0$,

$$
\begin{gathered}
y_{h} \triangleq y^{0} 1_{\{0\}}(t)+\frac{1}{h} \sum_{k=0}^{K-1}\left[(t-k h) y^{k+1}-(t-(k+1) h) y^{k}\right] 1_{(k h,(k+1) h]}(t) \\
\longrightarrow y \quad \text { strongly in } C\left([0, T] ; L^{2}(\Omega)\right) \cap H^{1}\left([0, T] ; H^{-1}(\Omega)\right), \quad(23)
\end{gathered}
$$

where $y$ is the solution of the system (1) with the limit control $u$ as above.

Remark 5. The above theorem contains two results: the uniform partial controllability and the convergence of the controls and states as $h \rightarrow 0$. The proof is standard. Indeed, the partial controllability statement follows from Theorem 2 and classical duality arguments [6]; while for the convergence result, one may use the approach developed in [17].

It is important to note that, in the limit, one can recover the controllability of (1) for all $T>2 R$, i.e. the same results as the multiplier method applied directly to the time-continuous wave equation yields, as we have shown in the last two properties of Theorem 3. Indeed, given any $T>2 R$, one can choose a sufficiently small $\delta$ such that Theorem 3 guarantees the controllability of the projections $\pi_{0, \delta h^{-2}}$ in time $T$. Since these projections involve the frequencies $\mu_{j}^{2}$ such that $\mu_{j}^{2}<\delta h^{-2}$, it is clear that, as $h \rightarrow 0$, this range of frequencies eventually covers the whole spectrum of the time-continuous wave equation. It is, however, important to underline that the filtering parameter $\delta$ has to be chosen depending on the value of $T$ and that $\delta \rightarrow 0$ as $T$ approaches $2 R$, as indicated in Remark 2.

By duality, Theorem 3 is a consequence of Theorem 2. Hence, in the sequel we shall concentrate mainly on the proof of Theorem 2. To show Theorem 2 , we shall develop a multiplier approach, which is a discrete analogue of the classical one for the time-continuous case [6]. There are two key ingredients when doing this. One is a basic identity for the solutions of (9) obtained by means of multipliers, which is a discrete version of the classical one on the time-continuous wave equation [6]. The other one is the construction of the filtering operator to guarantee the uniform observability of (9) after filtering. We shall explain them in more detail later in this paper. 


\section{Bicharacteristic Rays and Group Velocity}

Before entering into the details of the proofs, we give an heuristic explanation of the necessity of the above filtering mechanism in terms of the group velocity of propagation of the solutions of the time-discrete system (see $[12,17])$. For doing that we consider the time-discrete wave equation (9) in the whole space $\mathbb{R}^{d}$. Applying the Fourier transform (the continuous one in space and the discrete one in time), we deduce that the symbol of the time semi-discrete system (9) is

$$
p_{h}(\tau, \xi)=-\frac{4 \sin ^{2} \frac{\tau h}{2}}{h^{2}}+|\xi|^{2} \cos (\tau h), \quad(\tau, \xi) \in\left[-\frac{\pi}{2 h}, \frac{\pi}{2 h}\right] \times \mathbb{R}^{d}
$$

It is easy to see that, for all $\tau \in\left[-\pi(2 h)^{-1}, \pi(2 h)^{-1}\right], p_{h}(\tau, \xi)$ has two nontrivial roots $\xi^{ \pm} \in \mathbb{R}^{d}$. The bicharacteristic rays are defined as the solutions of the following Hamiltonian system:

$$
\left\{\begin{aligned}
\frac{d x(s)}{d s} & =2 \xi \cos (\tau h), & \frac{d t(s)}{d s} & =-\frac{2 \sin (\tau h)}{h}-|\xi|^{2} h \sin (\tau h), \\
\frac{d \xi(s)}{d s} & =0, & \frac{d \tau(s)}{d s} & =0 .
\end{aligned}\right.
$$

As in the continuous case, the rays are straight lines. However, both the direction and the velocity of propagation of the rays in this time-discrete setting case are different from the time-continuous one.

Let us now, for instance, illustrate the existence of bicharacteristic rays whose projection on $\mathbb{R}^{d}$ propagates at a very low velocity or even does not move at all. For this, we fix any $x_{0}=\left(x_{0,1}, \ldots, x_{0, d}\right) \in \Omega$ and choose the initial time $t_{0}=0$. Also, we choose the initial microlocal direction $\left(\tau_{0}, \xi_{0}\right)=$ $\left(\tau_{0}, \xi_{0,1}, \ldots, \xi_{0, d}\right)$ to be a root of $p_{h}$, i.e.,

$$
\left|\xi_{0}\right|^{2}=\frac{4 \sin ^{2} \frac{\tau_{0} h}{2}}{h^{2} \cos \left(\tau_{0} h\right)}, \quad \tau_{0} \in\left(-\frac{\pi}{2 h}, \frac{\pi}{2 h}\right) .
$$

Note that the above condition is satisfied for $\xi_{0,1}=2 h^{-1} \sin \frac{\tau_{0} h}{2} \cos ^{-1 / 2}\left(\tau_{0} h\right)$ and $\xi_{0,2}=\cdots=\xi_{0, d}=0$, for instance. In this case we get

$$
\frac{d x}{d t}=\frac{d x / d s}{d t / d s}=-\frac{\cos ^{3 / 2}\left(\tau_{0} h\right)}{\cos \frac{\tau_{0} h}{2}}
$$

and $d x_{2}(t) / d t=\cdots=d x_{d}(t) / d t=0$. Thus, $x_{j}(t)$ for $j=2, \ldots, d$ remain constant and

$$
x_{1}(t)=x_{0,1}-t \cos ^{3 / 2}\left(\tau_{0} h\right) \cos ^{-1} \frac{\tau_{0} h}{2}
$$

evolves with speed $-\cos ^{3 / 2}\left(\tau_{0} h\right) \cos ^{-1} \frac{\tau_{0} h}{2}$, which tends to 0 when $\tau_{0} h \rightarrow \frac{\pi}{2}-$, or $\tau_{0} h \rightarrow-\frac{\pi}{2}+$. This allows us to show that, as $h \rightarrow 0$, there exist rays that 


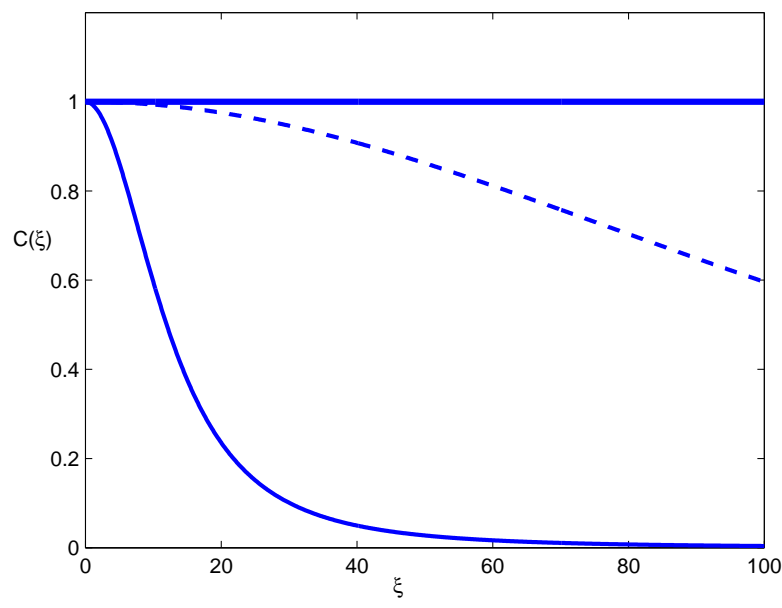

Fig. 1. The diagram of the group velocity $C(\xi) . h=0.1$ (solid line) vs. $h=0.01$ (dashed line). The thick horizontal segment corresponds to the theoretical group velocity $C(\xi)=1$ (in the continuous case, i.e. for $h=0$ ).

remain trapped on a neighborhood of $x_{0}$ for time intervals of arbitrarily large length. In order to guarantee the boundary observability, these rays have to be cut-off by filtering. This can be done by restricting the Fourier spectrum of the solution to the range $|\tau| \leq \frac{\rho \pi}{2 h}$ with $0<\rho<1$. This corresponds to

$$
|\xi|^{2} \leq \frac{4 \sin ^{2}(\rho \pi / 2)}{h^{2} \cos (\rho \pi / 2)}
$$

for the root of the symbol $p_{h}$.

This is the same scaling of the filtering operators we imposed on Theorems 2 and 3 , namely, $\mu_{j}^{2} \leq \delta / h^{2}$. Note, however, that, in (24), as $\rho \rightarrow 1$, the filtering parameter

$$
\delta=\frac{4 \sin ^{2}(\rho \pi / 2)}{\cos (\rho \pi / 2)} \longrightarrow \infty .
$$

Thus, in principle, as mentioned above, the analysis of the velocity of propagation of bicharacteristic rays does not seem to justify the need of letting the filtering parameter $\delta$ small enough as in Theorems 2 and 3. Thus, this last restriction seems to be imposed by the rigidity of the method of multipliers rather than by the underlying wave propagation phenomena.

We can reach similar conclusions by analyzing the behavior of the so-called group velocity. Indeed, following [12], in $1-d$ the group velocity has the form

$$
C(\xi)=\frac{4}{\left(2+h^{2} \xi^{2}\right) \sqrt{4+h^{2} \xi^{2}}},
$$


with the graphs as in Figure 1. Obviously, it tends to zero when $h^{2} \xi^{2}$ tends to infinity. This corresponds precisely to the high frequency bicharacteristic rays constructed above for which the velocity of propagation vanishes. Based on this analysis one can show that, whatever the filtering parameter $\delta$ is, uniform observability requires the observation time to be large enough with $T(\delta) \nearrow \infty$ as $\delta \nearrow \infty$. This may be done using an explicit construction of solutions concentrated along rays (see, for instance, [7]). The positive counterpart of this result guaranteeing that, for any value of the filtering parameter $\delta>0$, uniform observability/controllability holds for sharp large enough values of time, is an interesting open problem whose complete solution will require the application of microlocal analysis tools. At this respect it is worth mentioning that, although the results in [2] can be applied for any $\delta>0$, the value of the time they yield is larger than the one predicted by the analysis in this section.

\section{A Key Identity via Multipliers}

In this section we present the first key point of the proof of Theorem 2, i.e., an identity for the solutions of (9).

The desired identity is as follows:

Lemma 1. For any $h>0$ and any solution $\left\{\varphi^{k}\right\}_{k=0, \ldots, K}$ of (9), it holds

$$
\begin{array}{r}
\frac{h}{2} \sum_{k=0}^{K-1} \int_{\Omega}\left(\left\|\frac{\varphi^{k+1}-\varphi^{k}}{h}\right\|^{2}+\frac{\left|\nabla \varphi^{k+1}\right|^{2}+\left|\nabla \varphi^{k}\right|^{2}}{2}\right) d x+X+Y+Z \\
=\frac{h}{2} \sum_{k=1}^{K-1} \int_{\Gamma}\left(x-x_{0}\right) \cdot \nu\left\|\frac{\partial}{\partial \nu}\left(\frac{\varphi^{k+1}+\varphi^{k-1}}{2}\right)\right\|^{2} d \Gamma,
\end{array}
$$

where

$$
\begin{gathered}
X=\int_{\Omega}\left[\left(x-x_{0}\right) \cdot \nabla\left(\frac{\varphi^{K}+\varphi^{K-2}}{2}\right)+\frac{d-1}{2} \varphi^{K}\right] \frac{\varphi^{K}-\varphi^{K-1}}{h} d x \\
-\int_{\Omega}\left[\left(x-x_{0}\right) \cdot \nabla\left(\frac{\varphi^{2}+\varphi^{0}}{2}\right)+\frac{d-1}{2} \varphi^{0}\right] \frac{\varphi^{1}-\varphi^{0}}{h} d x \\
Y=\frac{d}{2}\left[h^{2} \sum_{k=1}^{K-1} \int_{\Omega} \Delta\left(\frac{\varphi^{k+1}+\varphi^{k-1}}{2} \frac{\varphi^{k}-\varphi^{k-1}}{h}\right) d x\right. \\
\left.\quad-h \int_{\Omega}\left\|\frac{\left.\varphi^{K}-\varphi^{K-1} \|^{2} d x\right]}{h}\right\|^{2}\right] \\
+\int_{\Omega}\left(x-x_{0}\right) \cdot\left[\nabla\left(\frac{\varphi^{K-1}-\varphi^{K-2}}{2}\right) \frac{\varphi^{K}-\varphi^{K-1}}{h}\right. \\
\left.+\nabla\left(\frac{\varphi^{2}-\varphi^{1}}{2}\right) \frac{\varphi^{1}-\varphi^{0}}{h}\right] d x
\end{gathered}
$$




$$
\begin{aligned}
Z=\frac{(d-2) h}{8} \sum_{k=1}^{K-1} \int_{\Omega}\left\|\nabla\left(\varphi^{k+1}-\varphi^{k-1}\right)\right\|^{2} d x \\
\quad-\frac{(d-1) h}{4} \sum_{k=0}^{K-1} \int_{\Omega}\left\|\nabla\left(\varphi^{k+1}-\varphi^{k}\right)\right\|^{2} d x \\
-\frac{(d-1) h}{4} \int_{\Omega}\left(\nabla \varphi^{K} \cdot \nabla \varphi^{K-1}+\nabla \varphi^{1} \cdot \nabla \varphi^{0}\right) d x \\
+\frac{(d-2) h}{4} \int_{\Omega}\left(\left|\nabla \varphi^{K-1}\right|^{2}+\left|\nabla \varphi^{1}\right|^{2}\right) d x .
\end{aligned}
$$

Proof. Multiplying the first equation of $(9)$ by $\left(x-x_{0}\right) \cdot \nabla\left(\varphi^{k+1}+\varphi^{k-1}\right) / 2$ (which is a discrete version of the classical multiplier $\left(x-x_{0}\right) \cdot \nabla \varphi$ for the wave equation), integrating it in $\Omega$, summing it up from 1 to $K-1$ and using integration by parts, we obtain

$$
\begin{aligned}
& h \sum_{k=1}^{K-1} \int_{\Omega}\left(x-x_{0}\right) \cdot \nabla\left(\frac{\varphi^{k+1}+\varphi^{k-1}}{2}\right) \frac{\varphi^{k+1}+\varphi^{k-1}-2 \varphi^{k}}{h^{2}} d x \\
& \quad=h \sum_{k=1}^{K-1} \int_{\Omega}\left(x-x_{0}\right) \cdot \nabla\left(\frac{\varphi^{k+1}+\varphi^{k-1}}{2}\right) \Delta\left(\frac{\varphi^{k+1}+\varphi^{k-1}}{2}\right) d x .
\end{aligned}
$$

One can check that the left hand side term of (29) coincides with

$$
\begin{aligned}
& \frac{d}{2} h \sum_{k=0}^{K-1} \int_{\Omega}\left\|\frac{\varphi^{k+1}-\varphi^{k}}{h}\right\|^{2} d x+Y \\
+ & \int_{\Omega}\left(x-x_{0}\right) \cdot \nabla\left[\left(\frac{\varphi^{K}+\varphi^{K-2}}{2}\right) \frac{\varphi^{K}-\varphi^{K-1}}{h}-\left(\frac{\varphi^{2}+\varphi^{0}}{2}\right) \frac{\varphi^{1}-\varphi^{0}}{h}\right] d x,
\end{aligned}
$$

where $Y$ is defined as in (27). We now use the classical multiplier identity for the Laplacian

$$
\int_{\Omega}\left(x-x_{0}\right) \cdot \nabla \psi \Delta \psi d x=\frac{1}{2} \int_{\Gamma}\left(x-x_{0}\right) \cdot \nu\left\|\frac{\partial \psi}{\partial \nu}\right\|^{2} d \Gamma-\frac{2-d}{2} \int_{\Omega}|\nabla \psi|^{2} d x
$$

which holds for all $\psi \in H^{2} \cap H_{0}^{1}(\Omega)$ [6]. Then, using the identity $(a+b)^{2}=$ $2\left(a^{2}+b^{2}\right)-(a-b)^{2}$ for any $a, b \in \mathbb{R}$, the right-hand side term of (29) may be written as 


$$
\begin{aligned}
& \frac{h}{2} \sum_{k=1}^{K-1} \int_{\Gamma}\left(x-x_{0}\right) \cdot \nu\left\|\frac{\partial}{\partial \nu}\left(\frac{\varphi^{k+1}+\varphi^{k-1}}{2}\right)\right\|^{2} d \Gamma \\
& \quad+\frac{(d-2) h}{2}\left\{\sum_{k=0}^{K-1} \int_{\Omega} \frac{\left|\nabla \varphi^{k+1}\right|^{2}+\left|\nabla \varphi^{k}\right|^{2}}{2} d x\right. \\
& \left.\quad-\sum_{k=1}^{K-1} \int_{\Omega}\left\|\nabla\left(\frac{\varphi^{k+1}-\varphi^{k-1}}{2}\right)\right\|^{2} d x-\frac{1}{2} \int_{\Omega}\left(\left|\nabla \varphi^{K-1}\right|^{2}+\left|\nabla \varphi^{1}\right|^{2}\right) d x\right\} .
\end{aligned}
$$

On the other hand, multiplying the first equation of (9) by $\varphi^{k}$ (which is a discrete version of the multiplier $\varphi$ in the time-continuous setting, which allows establishing the identity of equipartition of energy), integrating it in $\Omega$, summing it up for $k=1, \ldots, K-1$ and using integration by parts, as above, we obtain the following equipartition of energy identity:

$$
\begin{aligned}
& h \sum_{k=0}^{K-1} \int_{\Omega}\left(\left\|\frac{\varphi^{k+1}-\varphi^{k}}{h}\right\|^{2}-\frac{\left|\nabla \varphi^{k+1}\right|^{2}+\left|\nabla \varphi^{k}\right|^{2}}{2}\right) d x \\
& =-\frac{h}{2} \sum_{k=0}^{K-1} \int_{\Omega}\left\|\nabla\left(\varphi^{k+1}-\varphi^{k}\right)\right\|^{2} d x-\frac{h}{2} \int_{\Omega}\left(\nabla \varphi^{K} \cdot \nabla \varphi^{K-1}+\nabla \varphi^{1} \cdot \nabla \varphi^{0}\right) d x \\
& +\int_{\Omega}\left(\frac{\varphi^{K}-\varphi^{K-1}}{h} \varphi^{K}-\frac{\varphi^{1}-\varphi^{0}}{h} \varphi^{0}\right) d x
\end{aligned}
$$

By (29)-(33), recalling (26) and (28) respectively for $X$ and $Z$, we arrive at the desired identity $(25)$.

Remark 6 . The identity (25) is a time-discrete analogue of the following wellknown identity for the wave equation (9) obtained by multipliers [6]:

$$
\frac{1}{2} \int_{0}^{T} \int_{\Omega}\left[\left|\varphi_{t}\right|^{2}+|\nabla \varphi|^{2}\right] d x d t+\mathcal{X}=\frac{1}{2} \int_{0}^{T} \int_{\Gamma}\left(x-x_{0}\right) \cdot \nu\left\|\frac{\partial \varphi}{\partial \nu}\right\|^{2} d \Gamma d t
$$

where

$$
\mathcal{X}=\int_{\Omega}\left[\left(x-x_{0}\right) \cdot \nabla \varphi+\frac{d-1}{2} \varphi\right] \varphi_{t} d x \|_{t=0}^{T} .
$$

There are clear analogies between (1) and (34). In fact the only major differences are that, in the discrete version (1), two extra reminder terms ( $Y$ and $Z$ ) appear, which are due to the time discretization. It is easy to see, formally, that $Y$ and $Z$ tend to zero as $h \rightarrow 0$. But this convergence does not hold uniformly for all solutions. Consequently, these added terms impose the need of using filtering of the high frequencies to obtain observability inequalities out of (1) and modify the observability time, as we shall see. 


\section{Filtering and Uniform Observability}

In this section, we present the second key ingredient of the proof of Theorem 2 , i.e., the choice of the filtering parameter which, combined with the identity in Lemma 1, leads to the desired uniform observability inequality in Theorem 2.

For this, we first derive the following result, which provides an estimate on the reminder term $X+Y+Z$ in Lemma 1 in terms of the energy:

Lemma 2. Let $K$ be an even integer, $s>0$ and $T>0$. Then, for any $\left(\varphi^{0}, \frac{\varphi^{1}-\varphi^{0}}{h}\right) \in \mathcal{C}_{1, s} \times \mathcal{C}_{0, s}$, for the corresponding solution $\left\{\varphi^{k}\right\}_{k=0, \ldots, K}$ of (9), it holds

$$
X+Y+Z \geq-\left[2 R+a_{1} h+3 R \sqrt{s} h+T\left(\frac{d}{2} \sqrt{s} h+a_{2} s h^{2}\right)\right] E_{h}^{0},
$$

where

$$
a_{1}=3 d-2+\max \left(\frac{d-1}{2}, 2\right), \quad a_{2}=\min \left(1,(2-d)^{+}\right)+\frac{d-1}{2} .
$$

Proof. For any $\left(\varphi^{0}, \frac{\varphi^{1}-\varphi^{0}}{h}\right) \in \mathcal{C}_{1, s} \times \mathcal{C}_{0, s}$, in view of the Fourier series decomposition of the corresponding solution $\left\{\varphi^{k}\right\}_{k=0, \ldots, K}$ of $(9)$, one sees that, for any $k$, we have

$$
\begin{gathered}
\int_{\Omega}\left|\nabla\left(\varphi^{k}-\varphi^{k-1}\right)\right|^{2} d x \leq s \int_{\Omega}\left|\varphi^{k}-\varphi^{k-1}\right|^{2} d x \\
\int_{\Omega}\left|\Delta\left(\frac{\varphi^{k+1}+\varphi^{k-1}}{2}\right)\right|^{2} d x \leq s \int_{\Omega}\left|\nabla\left(\frac{\varphi^{k+1}+\varphi^{k-1}}{2}\right)\right|^{2} d x .
\end{gathered}
$$

Recalling (26)-(28) and using (37), and noting $T=K h$ and that the energy of the system (9) is conservative, we can show that

$$
\begin{gathered}
|X| \leq[2 R+2(d-1) h+R h \sqrt{s}] E_{h}^{0},|Y| \leq h\left[d\left(\frac{\sqrt{s} T}{2}+1\right)+2 R \sqrt{s}\right] E_{h}^{0}, \\
Z \geq-h\left\{\left[\min \left(1,(2-d)^{+}\right)+\frac{d-1}{2}\right] \operatorname{sh} T+\max \left(\frac{d-1}{2}, 2\right)\right\} E_{h}^{0},
\end{gathered}
$$

which gives (35).

Finally, Theorem 2 follows from Lemmas 1 and 2 immediately. Indeed, combining (25) and (35) and recalling the definition of $\Gamma_{0}$ in (2) we deduce that

$$
\begin{aligned}
\left\{T\left(1-\frac{d}{2} \sqrt{s} h-a_{2} s h^{2}\right)-\right. & {\left.\left[2 R+a_{1} h+3 R \sqrt{s} h\right]\right\} E_{h}^{0} } \\
\leq & \frac{R}{2} h \sum_{k=1}^{K-1} \int_{\Gamma_{0}}\left\|\frac{\partial}{\partial \nu}\left(\frac{\varphi^{k+1}+\varphi^{k-1}}{2}\right)\right\|^{2} d \Gamma_{0} .
\end{aligned}
$$


For this inequality to yield an estimate on $E_{h}^{0}$ we need to choose $s=\delta h^{-2}$ with $h$ small enough such that

$$
a_{2} \delta+\frac{d}{2} \sqrt{\delta}<1,
$$

or, more precisely,

$$
0<\sqrt{\delta}<\frac{4}{\sqrt{d^{2}+16 a_{2}}+d} .
$$

Once this is done, for $h \in\left(0, h_{0}\right), T$ has to be chosen such that

$$
T>\frac{2 R+a_{1} h_{0}+3 R \sqrt{\delta}}{1-\frac{d}{2} \sqrt{\delta}-a_{2} \delta} \geq 2 R .
$$

Hence, (20) holds for $h \in\left(0, h_{0}\right]$.

Conversely, for any $T>2 R$ one can always choose $h_{0}$ and $\delta$ small enough so that (38) and (39) hold, guaranteeing the uniform observability inequality (20).

\section{Further Comments and Open Problems}

Fully discrete schemes

The analysis in this paper can be combined with previous works (see, for instance, [17]) concerning space semi-discretizations to deal with full discretization schemes. This has been done in [2] in a more abstract setting. But a complete analysis of this issue is still to be done.

\section{Other equations}

The approach and results in this paper can be extended to other PDEs of conservative nature such as Schrödinger, plate, Maxwell's equations, and so on. There is a fruitful literature on the use of multiplier techniques for these models in the continuous setting (see, for instance, [6]). But, the analysis of the corresponding time-discrete systems, adapting the techniques developed in this paper, remains to be done.

\section{Variable coefficients and nonlinear problems}

It is well-known that, in the continuous case, the multiplier approach can be applied to obtain the controllallability/observability of the conservative PDEs with constant coefficients. As for the problems with variable coefficients and/or the nonlinear ones, one has to use microlocal analysis [1] and/or Carleman estimates [13] to get sharp results. In this time-discrete setting, it would be interesting to develop these other approaches to cover the same class of models as in the PDE setting. This is still to be done. 
Acknowledgement. This work is supported by the Grant MTM2005-00714 of the Spanish MEC, the project MTM2008-03541 of the Spanish Ministry of Science and Innovation, the DOMINO Project CIT-370200-2005-10 in the PROFIT program of the MEC (Spain), the i-MATH project of Spanish MEC, the SIMUMAT projet of the CAM (Spain), the NSFC under grants 10831007 and 60821091, and the NSF of China under grant 10525105.

\section{References}

1. C. Bardos, G. Lebeau, and J. Rauch. Sharp sufficient conditions for the observation, control, and stabilization of waves from the boundary. SIAM J. Control Optim., 30(5):1024-1065, 1992.

2. S. Ervedoza, C. Zheng, and E. Zuazua. On the observability of time-discrete linear conservative systems. J. Funct. Anal., 254(12):3037-3078, 2008.

3. G. Glowinski. Ensuring well-posedness by analogy: Stokes problem and boundary control for the wave equation. J. Comput. Phys., 103(2):189-221, 1992.

4. R. Glowinski, C. H. Li, and J.-L. Lions. A numerical approach to the exact boundary controllability of the wave equation. I. Dirichlet controls: description of the numerical methods. Japan J. Appl. Math., 7(1):1-76, 1990.

5. J. A. Infante and E. Zuazua. Boundary observability for the space semidiscretizations of the 1-D wave equation. M2AN Math. Model. Numer. Anal., 33(2):407-438, 1999.

6. J.-L. Lions. Contrôlabilité exacte, perturbations et stabilisation de systèmes distribués, Vol. 1. Masson, Paris, 1988.

7. F. Macià. The effect of group velocity in the numerical analysis of control problems for the wave equation. In Mathematical and Numerical Aspects of Wave Propagation - WAVES 2003, pages 195-200, Berlin, 2003. Springer.

8. M. Negreanu and E. Zuazua. Convergence of a multigrid method for the controllability of a 1-d wave equation. C. R. Math. Acad. Sci. Paris, 338(5):413-418, 2004 .

9. A. Osses. A rotated multiplier applied to the controllability of waves, elasticity, and tangential Stokes control. SIAM J. Control Optim., 40(3):777-800, 2001.

10. K. Ramdani, T. Takahashi, G. Tenenbaum, and M. Tucsnak. A spectral approach for the exact observability of infinite-dimensional systems with skewadjoint generator. J. Funct. Anal., 226(1):193-229, 2005.

11. J. W. Thomas. Numerical partial differential equations: Finite difference methods. Springer, Berlin, 1995.

12. L. N. Trefethen. Group velocity in finite difference schemes. SIAM Rev., 24(2):113-136, 1982.

13. X. Zhang. Explicit observability estimate for the wave equation with potential and its application. R. Soc. Lond. Proc. Ser. A Math. Phys. Eng. Sci., 456(1997):1101-1115, 2000.

14. X. Zhang, C. Zheng, and E. Zuazua. Time discrete wave equations: Boundary observability and control. Discrete Contin. Dyn. Syst., 23(1-2):571-604, 2009.

15. C. Zheng. Controllability of the time discrete heat equation. Asymptot. Anal., 59(3-4):139-177, 2008.

16. E. Zuazua. Boundary observability for the finite-difference space semidiscretizations of the 2-D wave equation in the square. J. Math. Pures Appl., 78(5):523-563, 1999. 
17. E. Zuazua. Propagation, observation, and control of waves approximated by finite difference methods. SIAM Rev., 47(2):197-243, 2005. 